\title{
GENOMIC PLASTICITY AND INFORMATION PROCESSING BY TRANSCRIPTION COREGULATORS
}

\author{
Arndt BENECKE
}

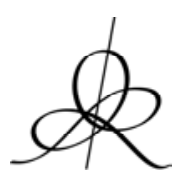

Institut des Hautes Études Scientifiques

35 , route de Chartres

91440 - Bures-sur Yvette

Décembre 2002

IHES/ M/ 02/ 96

To appear in ComPlexUs 


\title{
Genomic plasticity and information processing by transcription coregulators.
}

\author{
Arndt BENECKE \\ Institut des Hautes Études Scientifiques - 35, route de Chartres, 91440 Bures-sur-Yvette, France \\ INSERM U417 - 184, rue du Faubourg St.-Antoine, 75012 Paris, France
}

Journal: $\quad$ ComPlexUs

Type of paper: Review/Original Research

Running Title: The Coregulator Model of Transcription

Keywords: functional genomics, transcription regulation, coregulator, gene regulatory network, complex search expression, optimization

Correspondence: $\quad$ phone: + 33 (0)1 609266 65; fax: + 33 (0)1 609266 09; e-mail: arndt@ihes.fr 


\begin{abstract}
Functional genomics in higher eukaryotes faces major challenges attributable to the ever-increased complexity of the regulatory circuitry and its biological manifestation. The genomic regulon remains largely inaccessible to bioinformatics tools due to a degree of apparent functional redundancy (degeneration) of the underlying code and nonlinearities of its cellular interpretation.

Based on recent biochemical work on transcription coregulators and their crosstalk with other genome maintenance and regulation pathways a model is developed which offers an explanation to how the cellular transcription machinery interprets the gene regulatory code. The experimental evidence suggests a secondary layer of coding by the chromatin structure of the genome producing hypercycles of repressed and open nucleosomal sites in euchromatin. The code is read-out by coregulators analog to and prior to the reading-out of the primary coding (the nucleotide sequence) by transcription factors. The coregulator model of transcription thus promises not only to enhance our comprehension of the biological phenomena leading to faithful gene regulation in higher eukaryotes, but also to overcome some of above described impediments to bioinformatic analysis, and lead to the systematic description of gene regulatory networks and their dynamics.

The implications of a coregulator-based view of transcription for the development of tools analyzing genomes of higher eukaryotes for complex, composite nucleotide patterns derived from above molecular understanding are significant. Furthermore, the recent generation of systematic gene expression information in response to specific cellular signals can be utilized within limitations as training data in multiple-round feed-back optimization of these complex search expressions.
\end{abstract}

Keywords: functional genomics, transcription regulation, coregulator, gene regulatory network, complex search expression, optimization. 


\section{Introduction}

Recent progress in molecular biology and physical chemistry has led to the generation of systematic sequence and gene expression information for genomes of higher eukaryotes including man [1, 2]. Tools such as spatio-temporal controlled gene-inactivation in mice [3], the establishment of in-vitro accessible mammalian development and cell differentiation models [4], and the biochemical characterization of their signaling and gene expression pathways have been developed [5]. A successful combination of these methods will allow for the first time to render genome regulatory circuits accessible to more fundamental mathematical analysis of information processing, stability, emergence, and self-adaptation in higher eukaryotes.

The activity of all gene regulatory circuits is a direct reflection of the integration of the cellular state ${ }^{1}$ at any given time. In response to intra- and extra-cellular signals the activity of gene regulatory circuits is under constant change, thereby supplying the cell with the required set and amounts of all gene products to act in accordance with the signaling event. The main regulatory load of this immensely complex autoexpression of the genome is carried by the process of gene transcription, the first step in gene expression [6, and therein].

\section{Gene Transcription and its Regulation.}

The vast majority of genes are in their expression under tight control and vary according to changes in the cellular requirements. Extra- and intra-cellular signals directly define positively and negatively the expression levels of target genes and by consequence assemble specific gene programs. Although any gene within the program can be regulated by many different signals, composition and activity of the entity is specific to its signal and both thus reciprocally defined. The regulation of these gene programs has to be tight for obvious reasons [6].

The process of gene transcription is achieved by a machinery of great complexity consisting of a DNA dependent RNA polymerase and associated general transcription factors (GTFs) - the basal transcription machinery. In higher eukaryotes the composition of sub-complexes within the basal machinery is dynamic and the total amount of factors participating is within the range of approximately fifty to over one-hundred.

\footnotetext{
${ }^{1}$ Defined as the ensemble of all incoming signals, their chemical manifestation, and the actual physical/molecular composition of the cell.
} 
The dynamic assembly of these complexes in higher eukaryotes is controlled, and represents a first level of regulation [6].

The second and predominant level of transcription control is achieved through the use of DNA sequence specific transcription factors that activate or repress transcription when bound to the control region of the corresponding target gene. The utilization of sequence-specific transcription factors constitutes a multitude of regulatory degrees of freedom of which the most important are the presence and accessibility of transcription factor binding sites, and the availability and activity of the corresponding transcription factors and their coregulators [6].

The complete set of sequence specific and unspecific transcription factors together with their coregulators determine the expression level of a target gene through the integration of the different cellular signals they respond to. The entity of all transcription factors, their target sequences, and their response-capacity to all signals that can be integrated is the genomic regulon of an organism. We estimate the number of degrees of freedom of any genomic regulon to exceed by large the number of genes $x$ the number of cell-types $x$ the number of signals [6].

\section{Genome Structure and its Impact on Transcription Regulation.}

Especially in eukaryotes, as opposed to prokaryotes, the packaging of DNA into chromatin not only represents an essential organizational but also an important regulatory feature. Chromatin structure imposes a direct obstacle to the process of gene transcription, and can specifically be modified to contribute positively or negatively to the correct assembly of transcription factors and their activity $[6,7]$. Figure 1 illustrates the shift in paradigm from prokaryotes that mainly regulate the expression of target genes through GTF recruitment towards a much more chromatin dependent regulation in eukaryotes.

Most importantly, a recent study has clearly demonstrated that chromatin/histone (the basic constituents of chromatin) modifications are not only required for the de-repression step in gene activation but are also a necessary part in the activation process [8].

Hence, it is no surprise that the majority of regulation encompasses modification of the chromatin structure of, and around target genes [9]. The modifications which occur at the level of the chromosome, the nucleosomal array and the individual nucleosomes and the histones are ample, and only in part understood [10-13]. These modifications are orchestrated by the transcription factors and executed by the 
only recently discovered transcription coregulators [7]. Just as transcription factors, coregulators can act in a positive (coactivator) or negative (corepressor) manner upon the activity of a given transcription factor or set of transcription factors. Coregulators do not specifically interact with DNA (as opposed to sequencespecific transcription factors) but display some degree of gene specificity (as opposed to general transcription factors) [7]. Coregulators provide an essential link between gene specific regulators and cellular signaling and help to establish genomic plasticity. A schematic presentation of coregulator activity on gene expression and the physico-chemical processes it relies on are shown in Figure 2. Please refer to the legend for further explanations. 


\section{The Coregulator Model of Transcription}

The transcription apparatus of any given cell is capable to correctly read-out the regulatory information encoded in the sequence and integrate it with the intra- and extra-cellular signals which have instructive function upon its activity. The margin of error of this sophisticated machinery is orders of magnitude below the one of current bioinformatic analysis. The idea of a degenerative code and functional redundancy between transcription factors, as well as our current insight into coregulator function has prompted a coregulator-based view of transcription regulation, which is presented below.

\section{Nonlinearities in Transcription Signal Integration.}

Despite complete sequence information on an increasing number of eukaryotic genomes, the deciphering of the regulatory circuitry is tremendously complicated by nonlinearities in the integration of transcription signals. While transcription factor availability and activity can still simplistically be reduced to linear components in their contribution to the overall gene activity, the activity and interplay of coregulators can not. Several examples of our recent work are discussed to illustrate this fact. Among the most important biophysical effects of coregulators are the establishment of synergistic regulation [14, 15, 16, Figure 3] and cooperative DNA binding of transcription factors [17, Figure 3], switching between corepression and coactivation [18, 19, Figure 5], and cross-regulation with other DNA maintenance and replication processes [20, Figure 5].

Mechanisms of coregulator-induced transcription factor synergy and cooperative DNA binding. Synergy between transcription factors has been widely recognized as a predominant means to converge several different signals (encoded by specific transcription factors) upon the expression of a target gene. Synergy between transcription factors might be introduced through different mechanisms, which include direct interactions between the implicated transcription factors. In most cases promoter architecture does not seem to be permissible for such direct contacts between different transcription factors. The characterization of transcription coregulators and their multiple transcription factor interaction domains has prompted the hypothesis that they might be at the basis of synergistic activation between transcription factors that can not directly interact with each other. In order to assess such a possibility different transcription factor and coregulator mutants were employed and tested for their capacity to maintain 
synergy on composite promoters (a summary of data obtained through such experiments can be found in Figure 3 - left panel). It was indeed possible to show that (i) coregulators such as the nuclear receptor coregulator transcription intermediary factor-2 (TIF2) are capable of promoting synergy between isolated transcription activators $[14,15]$, (ii) that such a mechanism requires simultaneous interaction between the coregulator and both transcription activators [14, 15], and (iii) that such a mechanism can be shown to also operate on other transcription factors [15]. The demonstration of a molecular mechanism for coregulator-induced synergy $[14,15,16]$ has the potential to explain synergistic effects between different transcription factors at a much more global scale, an idea supported by recent progress $[15,16,21]$. Coregulators might induce synergy between transcription factors by distinct means. Figure 4 illustrates two alternate models that can explain the effect of coregulator-mediated synergy. In the first case the amount of coregulator activity at a promoter has to overcome a certain critical threshold (upper panel), which can only be achieved through concerted recruitment of the coregulator by all participating transcription factors (left side). Alternatively, coregulator activity has to overcome a spatial threshold (lower panel, i.e. an opened chromatin domain has to be established over a certain minimal length with respect to the DNA sequence). In this latter case a coregulator does not necessarily directly interact with the different transcription factors present at the promoter, but rather several coregulator molecules are independently recruited by the transcription factors covering an extended region of the regulatory sequence (left side). Interestingly, we believe that the synergy studied in $[14,15,16]$ rather corresponds to the first case presented; however, currently no experimental protocol allows to unequivocally distinguish between the two modes presented. Likely, cellular mechanisms profit from a melange between the different options [21].

Interestingly, in cases where the promoter architecture does permit direct transcription factor contacts, and cooperativity in the DNA binding of transcription factors can also be established or enhanced by coregulators leading to another mechanism by which coregulators introduce nonlinearity in the gene regulation process. The nuclear receptors retinoic acid receptor (RAR, in Figure 3 right panel 'A') and retinoic $\mathrm{X}$ receptor (RXR, 'B') can cooperatively bind a DNA regulatory site called direct repeat spaced by 5 nucleotides (DR5, in Figure 3: a grey and black box). While RAR ('A') can homodimerize on DR5 elements, RXR ('B') on its own is incapable of engaging with the DR5 element on its own. The coregulator 
thymine-DNA glycosylase (TDG) is capable of selectively enhancing DNA binding of RAR-RXR ('AB') heterodimers on the DR5 element [17, Figure 3 - right panel].

\section{Mechanisms of coregulator crosstalk.}

Nonlinearity of transcription regulatory read-out can also be observed at a higher order with coregulators influencing their respective activities. A drastic example of such a higher order nonlinearity is observed between the coregulators receptor interacting protein of 140kDa (RIP140, in Figure 5 - left panel: 'CoR1') and the TBP-free TAF containing complex (TFTC, here: 'CoR2'). Both are capable of interacting with the same transcription factor (here: 'A'), while RIP140 ('CoR1') strongly represses transcription when bound to the transcription factor alone [19], TFTC on its own does not have measurable activity [18]. However, if both coregulators are present TFTC converts RIP140 from a corepressor into a coactivator [18, 19, Figure 5 - left panel], inversing the 'output' of promoter activity from repression to strong synergistic activation.

Similarly, a complex of CREB binding protein (CBP, in Figure 5 -right panel: 'CoR2') and thymine DNA glycosylase (TDG, here: 'CoR1') is not only active in transcription but also DNA repair of G:T and G:U DNA base mismatches [20, Figure 5 - right panel]. Both factors exert cooperativity between each other and induce synergistic transcription activity on different transcription factor complexes [20, Figure 5 - right panel]. The cross-regulation of coregulator activity seems to be a general mechanism as for instance observed for SET-domain factors [22-26] or the regulation of TIF2-CBP coactivator activity by intracellular signaling cascades [27]. The involvement of the same coregulator pair in both base-excision repair and transcription effectively links both processes and opens the possibility of cross-regulation (or: crosstalk) between both DNA dependent machines [20]. It is asserted that such a crosstalk will greatly benefit both timing and spatial coordination of DNA repair and transcription in a way that transcriptionally active sites are preferentially repaired to assure fidelity of former process. Reminiscent of this crosstalk are several independent observations such as the cross-coupling of nucleotide excision repair with transcription [28], double-strand break repair with coregulator action [29], epigenetic regulation through DNA methylation/demethylation and transcription [30], replication coupled DNA repair [31], a link between transcription regulation and RNA splicing [32], and a mechanism rerouting the general transcription factor TFIID during apoptosis [33]. 


\section{Degeneration of the Regulatory Code.}

The reciprocal mapping of transcription factors and regulatory elements is - unless further information is considered - rendered impossible due to the degeneration of the regulatory sequence elements, and partial functional redundancy between closely related transcription factors [34]. The presence of a consensus (i.e. high affinity) regulatory element does not necessarily confer regulatory capacity to the corresponding DNA fragment. As a matter of fact, most transcription factor binding sites occur at mean statistic frequency (given as $\langle f\rangle=\left(\left(P_{A, T}\right)^{n}\left(P_{G, C}\right)^{m}\right)^{-1}$ with $n$ being the number of $A+T$ nucleotides $(m=G+C)$ of the regulatory element and $\mathrm{P}$ their probability of occurrence) within the genomes of higher eukaryotes. Thus for the human genome $\left(3 \times 10^{9}\right.$ base pairs $)$ a given 8 -mer regulatory element occurs $>5 \times 10^{4}-2 \times 10^{5}$ times - a number higher than the estimated total amount of genes $\left(4 \times 10^{4}\right)$. Since conservative estimates of the number of direct target genes of a given transcription factor are in the order of $10^{1}$ to $5 \times 10^{2}$ on the high end, clearly the majority of consensus motifs found is non-functional [35]. This is in striking contrast to prokaryotes where genomic complexity is much smaller. However, still noisy transcription due to a degenerate code is observed [36]. Furthermore, most transcription factors do not only bind to a single, defined regulatory sequence but also interact with reduced affinity with motifs derived thereof $[37,38]$. There are examples of transcription factors acting rather through low-affinity versus high-affinity consensus elements [i.e. 39]. We explain this observation with coregulator action that induces binding cooperativity between several transcription factors (compare above), and speculate that these phenomena exist to even further increase regulatory capacity. Finally, highly related transcription factors often display functional redundancy in the sense that one can - within limitations - substitute functionally for the other in case of a loss-of-function mutation [34]. Since these highly related factors even utilize identical or very closely related recognition sites there is currently no way to a priori pin down the transcription factor that acts through a given regulatory element or vice versa.

\section{The chromatin sequence code.}

Chromatin structure provides an active component of gene regulation for both repression [7] and activation [8]. Transcription factors are, according to current models of gene regulation in eukaryotes, thought to first engage with their recognition elements and then, upon activation, orchestrate the remodeling of chromatin to allow transcription to occur. In contradiction, experimental evidence indicates that activation of 
transcription factors might be prior to DNA engagement [40], and second that transcription factor binding to DNA, at least in certain cases, depends on a pre-opened chromatin [41], and might be independent of the transcription process [42]. This strongly suggests that DNA binding of transcription factors is not necessarily the first step in the sequence of events leading to transcription regulation.

These findings have led to propose here a model of how the transcription machinery reads out and processes regulatory information. The model introduces the concept of a secondary gene regulatory code which is carried by the histone-modifications (the 'chromatin sequence', Figure 6). The existence of such histone-modifications and their role within transcription regulation have been very well documented experimentally, and are accepted to play a major role in coregulator-driven processes [7]. New is rather the notion of a code implemented into the actual composition of these chromatin-modifications. We propose that coregulators which display an affinity for (un-)modified histone tails and core nucleosomes (the affinity of an enzyme, the coregulator, for its substrate, the histone-tail) specifically recognize particular 'chromatin-sequences', thereby increasing their local concentration around these sites, and inducing chromatin-breathing (through the interplay of corepressors and coactivators, see Figures 6 and 7 for further explanation). Such chromatin breathing has indeed been observed for instance in the case of the ATP-dependent chromatin remodeling machine SWI/SNF [43, 44, 45]. Transcription factors then in turn can access DNA at these sites of pre-opened chromatin, shift the equilibrium between the 'repressed' and the 'open' state towards the latter, and engage the transcription apparatus (Figure 7). Interestingly this model also allows incorporation of higher-order nucleosome packing (as found in heterochromatin) during i.e. epigenetic activation and repression [46] in a fashion similar to transcription activation, in that epigenetic regulatory factors (i.e. DNA methyltransferases) remove repressed ('[R]') nucleosomes from the coregulator maintained hypercycle (for theoretical background on hypercycles consult: [47]) and transform them to the condensed ('[C]') state (Figure 7, i.e. [48-51]). Such a symmetric model is especially appealing since it does almost naturally account for recent experimental evidence. For instance it has been established that epigenetic silencing in Arabidopsis is composed of two stages, just as the present model suggests for both the activation and repression process [52]. Moreover, coregulator complexes have been found to delineate sites of polycomb repression, an observation in full agreement with the model presented here, and only with difficulties to reconcile with previous models $[53,54,55]$. Furthermore, the chromatin-code model explains how boundaries between chromatin domains are established (through 
coregulator-induced breathing of nucleosomal domains that act as substrates for the coregulators), a problem frequently raised [i.e. 56, 57, 58]. Finally, the model shown in Figure 7 also can provide for an explanation of how transcription (and other sequence specific DNA-based processes that require the formation of [O] states) can rapidly be inactivated during i.e. chromosome condensation during mitosis: The onset of mitosis is among other features characterized by the high synthesis of linker histone ( $\mathrm{H} 1$, and $\mathrm{H} 1$-like) and other nucleosome condensing proteins. These proteins (here: 'ER') will drive the formation of [C] state nucleosomes by metabolizing the [R] state nucleosomes. Even with constant transcription factor ('TF') and coregulator ('CoA', 'CoR') concentrations diminished [R] concentration will lead to increased activity of corepressors and thus diminished concentration of [O] state nucleosomes. This in turn will prevent TF engagement with target sites and thus formation of $[\mathrm{A}]$ state nucleosomes through the reduced availability of $[\mathrm{O}]$ states (Figure 7).

The model suggests thus the evolution of a second gene regulatory code, which is implemented on top of the primary DNA-sequence based code. The appearance of chromatin and its ample modifications parallels (on a crude scale) the appearance of coregulators during evolution. It is proposed here that coregulators are to 'chromatin-sequence' what transcription factors are to DNA-sequence. Ample histone modifications have been reported to occur concomitant to transcription regulation, and have been correlated with both activation and repression [see Introduction, 7]. While histone acetylation and deacetylation has received widest attention so far, it is increasingly evident that also histone methylation leads an alternate route to transcription activation $[59,60]$. Since chromatin could be understood as an outer shell to the DNA which is wound up in this protein structure, chromatin-modification might well be the initial, and not as previous thought, a concomitant or subsequent step in gene activation. Obviously, 'chromatin-sequence' is likely to be dependent on the underlying DNA-sequence code or the activity exerted by the DNA sequence during different DNA-based sequence specific processes. It is for instance maintained that chromatin structure is cell and cell-stage specific $[61,62]$ including in euchromatic regions, thus providing further evidence for the model proposed here. However, features of chromatin-structure contribute to epigenetic regulation (i.e. telomeric and centromeric regions of the chromosomes) and directly link epigenetic regulation to the physiology of cancer $[63,64]$ and development [65]. Thus the secondary code proposed here is neither independent of, nor completely dependent on the underlying DNA-sequence. 
Finally, it is possible to deduce from experimental evidence in the context of above model that certain features of the DNA-sequence code can be predicted through interpretation of the constraints that faithful coregulator action imposes on the architecture of regulatory elements. In particular it is proposed that:

\section{(A) Sequence Constraints through Coregulator Requirement}

The regulatory sequence elements of a gene and incoming signaling events instruct the assembly in space and time of a higher-order complex consisting of coregulators and associated transcription factors. This complex is not necessarily static nor necessarily represents direct interactions between its individual components, but rather reflects orchestrated action of all its components. The chromatin structure of the target region plays a crucial role in the instruction (where) and assembly (when). By contrast to its individual components or sub-complexes thereof, it is solely competent to process the entire available and required information and compute the corresponding output.

\section{(B) Reciprocal Mapping of Complex Features and Signaling Events}

The individual transcription factor-coregulator complexes that act upon genes of a common gene programs share also common architectural and functional features beyond or despite their actual composition since they respond to the same signal. These features are encoded in a common DNAsequence architecture and a similar 'chromatin-sequence', and can principally be understood and used in analysis.

\section{(C) Signal Interpretation Capacity as Consequence of Complex Sequence Features}

Any given signal establishing a gene regulatory program can be mapped upon features of the regulatory sequence beyond primary transcription factor binding sites due to the constraints faithful coregulator action imposes on before chromatin domains.

It will be of outmost importance to develop experimental tools to attempt sequencing chromatinmodifications analogous to genome-sequencing. Furthermore, it is maintained that it should be possible - given the instructive role that DNA-sequence and its activity has on the chromatin-sequence, 
and thus on coregulator activity - to deduce from coregulator action architectural features of the transcription factor binding sites. 


\section{Discussion}

Recent research on eukaryotic transcription control has uncovered nonlinearities associated with the interpretation of regulatory sequence $[8,14-19]$ and put further emphasis on the degeneration of the underlying code conjunct with functional redundancy of the transcription factors [34]. Furthermore, there is compelling evidence to suggest a coregulator-based view of transcription-regulation, in which the initial layer of the code-interpretation machinery is rather made up of coregulators than transcription factors. These facts hamper the predictive quality of bioinformatics tools that do not incorporate a priori information beyond recognition sequences to analyze the genomes of higher eukaryotes. The coregulator model of transcription, presented here, can explain plasticity on the genomic level, and promises to overcome some of these impediments by providing a conceptual link between signaling events and features beyond the pure sequence of regulatory elements of genes belonging to the same gene regulatory network. By suggesting a secondary code implemented in the histone-tail modifications which is interpretated by transcription coregulators the different nonlinearities associated with coregulator action (as discussed in detail above) can be put on a formal ground. The euchromatin structure hence participates in the choice of the particular coregulator that is to be employed during the transcription regulation process. This, as demonstrated, will in turn help to associate different coregulators and their activities with specific domains of chromatin, analog to how we associate different transcription factors with specific DNA regulatory elements. The model predicts that coactivators ('CoA') will open chromatin at all nucleosomal sites that are a substrate for the specific CoA enzymatic activity (low specificity), whether they are used as sites of gene regulation or not. Only in a subsequent step (high specificity), through the engagement of a transcription factor ('TF'), a subset of these opened sites become sites of active transcription ('[A]'), while the remainder is being converted back to the $[R]$ state through the action of a corepressor ('CoR') with opposite activity as the CoA. By introducing coregulator prior to transcription factor action the comparatively low specificity of coregulators for histone tails is fully exploited by the cell (In the opposite case, after engagement of the highly specific transcription factor, no further specificity will be gained by the subsequent recruitment of the coregulator). This comes at the cost of the energy needed to maintain the hypercycle between $[\mathrm{R}]$ and $[\mathrm{O}]$ states. However, it furthermore also provides a mechanism of how the cell overcomes ambiguities due to the degenerate nature of the regulatory DNA sequence code. 
The implications for bioinformatic analysis are significant since this model implies the necessity to analyze entire gene regulatory networks in order to extract the required information ('chromatin-sequence', coregulator-imposed DNA-sequence constraints) to understand the regulation of any individual gene within the program. However, on the other hand it provides an initial formalism that will and can be exploited during a priori in silico analysis of genomes for their regulatory capacities. Furthermore, based on the idea of low-specificity coregulator-induced chromatin-breathing a more comprehensive model of transcription regulation in eukaryotes can be developed that will grasp the essential features and mechanisms of the initial phase of gene expression.

We have begun to test the feasibility of translating these insights and the hypothesis generated there from, into an algorithm A.C.E. (for: Annotation of Complex Enhancers) that for example analyzes the gene regulatory program induced by retinoic acid (the signal) in human hematopoiesis [66]. The availability of systematic gene expression profiles for normal and loss-of-function mutant cells can thereby be exploited to generate fitness functions for individual composite nucleotide patterns the algorithm uses as input [66]. This quality measure is being exploited for optimization of parameters and automated refinement of those patterns [66, 67]. Furthermore, algorithms such as S.M.A.S.H. that extract the regulatory regions from homologues genes from mouse and human [68] demonstrate that indeed chromatin-domain structure is conserved between both organisms [68] and hence in principal can be extracted based on the formalism presented here. 


\section{Acknowledgements}

The author is indebt to the members of the Cayre and Lutz (both: INSERM U417, France), Chambon (IGBMC, France), Gromov (IHES, France), Rajewsky (The Rockefeller University, U.S.A.), and Tini (London Regional Cancer Center, Canada) groups for the outstanding scientific collaborations that have generated some of the crucial experimental evidence this work was based upon. Furthermore, their shared insights, helpful comments and suggestions have greatly aided the development of the coregulator model of transcription, issue of present manuscript. The author regrets that due to space limitations many original articles have been cited only indirectly. The recent text-book [reference 6, Editor: Joseph Locker] reunites over 2500 original citations within a dozen chapters dealing in-dept with the different aspects of transcription regulation, and can but be recommended for further reading.

This work was supported by grants from the European Commission (TMR), the Fondation pour la Recherche Médicale, and the Deutsche Forschungsgemeinschaft. 


\section{References}

[1] Venter JC et al. (2001) The sequence of the human genome. Science 291:1304-51.

[2] Lockhart DJ, Winzeler EA (2000) Genomics, gene expression and DNA arrays. Nature 405:827-36.

[3] Mark M, Ghselnick NB, Wendling O, Dupe V, Mascarez B, Kastner P, Chambon P (1999) A genetic dissection of the retinoid signalling pathway in the mouse. Proc. Nutr. Soc. 8:609-13.

[4] de The H, Chelbi-Alix MK (2001) APL, a model disease for cancer therapies? Oncogene 20:7136-9.

[5] Lin RJ, Sternsdorf T, Tini M, Evans RM (2001) Transcription regulation in acute promyelocytic leukemia. Oncogene 20:7204-15.

[6] Transcription Factors, Editor: Joseph Locker (2000) BIOS Scientific Publishing, UK, ISBN: 0124543456

[7] Orphanides G, Reinberg D (2002) A unified theory of gene expression. Cell 108:439-51.

[8] An W, Pelhan VB, Karymov MA, Leuba SH, Roeder RG (2002) Selective requirements for histone $\mathrm{H} 3$ and $\mathrm{H} 4 \mathrm{~N}$ termini in p300-dependent transcriptional activation from chromatin. Mol. Cell 9:81121.

[9] Lee TI, Young RA (2000) Transcription of eukaryotic protein-coding genes. Annu. Rev. Genet. 34:77-137.

[10] Wolffe AP (1992) New insights into chromatin function in transcriptional control. FASEB J. 6:335461.

[11] Wolffe AP (2001) Transcriptional regulation in the context of chromatin structure. Essays Biochem. $37: 45-57$

[12] Tansey WP (2001) Transcriptional activation: risky business. Genes Dev. 15:1045-92.

[13] Georgel PT (2002) Chromatin structure of eukaryotic promoters: a changing perspective. Biochem. Cell Biol. 80:295-300.

[14] Benecke A, Chambon P, Gronemeyer H (2000) Synergy between estrogen receptor activation functions AF1 and AF2 mediated by transcription intermediary factor TIF2. EMBO R. 1:151-7.

[15] Bommer M, Benecke A, Gronemeyer H, Rochette-Egly C (2002) TIF2 mediates synergy between RARalpha1 activation functions AF-1 and AF-2. J. Biol. Chem. 277:37961-6. 
[16] Germain P, lyer J, Zechel C, Gronemeyer H (2002) Coregulator recruitment and the mechanism of retinoic acid receptor synergy. Nature 415:187-92.

[17] Um SJ, Harbers M, Benecke A, Pierrat B, Losson R, Chambon P (1998) Retinoic acid receptors interact physically and functionally with the T:G mismatch-specific Thymine-DNA glycosylase. J.Biol.Chem. 273:20728-36

[18] Benecke A, Gaudon C, Garnier JM, vom Baur E, Chambon P, Losson R (2002) ADA3-containing complexes associate with estrogen receptor alpha. Nucl.AcidsRes. 30:2508-14.

[19] Benecke A, Tini M, Lade-Nielsen A, Losson R, Chambon P (2002) Activation-Repression switching by nuclear receptor coregulators. in preparation.

[20] Tini M, Benecke A, Um SJ, Torchia J, Evans RM, Chambon P (2002) Association of CBP/p300 acetylase and Thymine DNA Glycosylase links DNA repair and transcription. Mol. Cell. 9:265-77.

[21] Maniatis T, Reed R (2002) An extensive network of coupling among gene expression machines. Nature 416:499-506.

[22] Yeates TO (2002) Structures of SET domain proteins: protein lysine methyltransferases make their mark. Cell 111:

[23] Trievel RC, Beach BM, Dirk LMA, Houtz RL, Hurley JH (2002) Structure and catalytic mechanism of a SET domain protein methyltransferase. Cell 111:

[24] Wilson JR, Jing C, Walker PA, Martin SR, Howell SA, Blackburn GM, Gamblin SJ, Xiao B (2002) Crystlal structure and functional analysis of the histone methylatransferase SET7/9. Cell 111:

[25] Zhang X, Tamaru H, Khan SI, Horton JR, Keefe LJ, Selker EU, Cheng X (2002) Structure of Neurospora SET domain protein DIM-5, a histone H3 lysine methyltransferase. Cell 111:

[26] Firestein R, Cui X, Huie P, Cleary ML (2002) Set domain-dependent regulation of transcriptional silencing and growth by SUV39H1, a mammalian ortholog of drosophila Su(var)3-9. Mol. Cell. Biol. 20:4900-9.

[27] Su LF, Wang Z, Garabedian MJ (2002) Regulation of GRIP1 and CBP coactivator activity by Rho GDI modulates estrogen receptor transcriptional enhancement. J. Biol. Chem. 277:37037-44.

[28] Ura K, Hayes JJ (2002) Nucleotide excision repair and chromatin remodeling. Eur. J. Biochem. 269:2288-93. 
[29] Bird AW, Yu DY, Pray-Grant MG, Qiu Q, Harmon KE, Megee PC, Grant PA, Mitchell Smith M, Christman MF (2002) Acetylation of histone H4 by Esa1 is required for DNA double-strand break repair. Nature 419:411-5.

[30] Detich N, Theberge J, Szyf M (2002) Promoter-specific activation and demethylation by MBD2/Demethylase. J. Biol. Chem. 277:35791-4.

[31] Ridgway P, Almouzni G (2000) CAF-1 and the inheritance of chromatin-states: at the crossroads of DNA replication and repair. J. Cell Sci. 113:2647-58.

[32] Auboeuf D, Honig A, Berget SM, O'Malley BW (2002) Coordinate regulation of transcription and splicing by steroid receptor coregulators. Science 298:416-9.

[33] Bell B, Scheer E, Tora L (2001) Identification of hTAFII80delta links apoptotic signaling pathways to transcription factor TFIID function. Mol. Cell 8:591-600.

[34] Morrison AD (1998) $1+1=r 4$ and much much more. Bioessays 20:794-7.

[35] Rajewsky N, Benecke A (2001) unpublished.

[36] Elowitz MB, Levine AJ, Siggia ED, Swain PS (2002) Stochastic gene expression in a single cell. Science 297:1183-6.

[37] Gerland U, Moroz JD, Hwa T (2002) Physical constraints and functional characteristics of transcription factor-DNA interactions. Proc. Natl. Acad. Sci. 99:12015-20.

[38] Liu X, Clarke ND (2002) Rationalization of gene regulation by a eukaryotic transcription factor: calculation of regulatory region occupancy from predicted binding affinities. J. Mol. Biol. 323:1-8.

[39] Wright WE, Funk DE (1993) CASTing for multicomponent DNA-binding complexes. Trends Biochem. Sci. 18:77-80.

[40] Young ET, Kacherovsky N, Van Riper K (2002) Snf1 protein kinase regulates Adr1 binding to chromatin but not transcriptional activation. J. Biol. Chem. 277:38095-103.

[41] Andrau JC, Van Oevelen CJC, Van Teeffelen HAAM, Weil PA, Holstege FCP, Timmers HTM (2002) Mot1p is essential for TBP recruitment to selected promoters during in vivo gene activation. EMBO J. 21:5173-83.

[42] Kuo MH, vom Baur E, Struhl K, Allis CD (2000) Gcn4 activator targets Gcn5 histone acetyltransferase to specific promoters independently of transcription. Mol. Cell 6:1309-20. 
[43] Aoyagi S, Hayes JJ (2002) hSWI/SNF-catalyzed nucleosome sliding does not occur soley via a twist-diffusion mechanism. Mol. Cell. Biol. 22:7484-90.

[44] Kassabov SR, Henry NM, Zofall M, Tsukiyama T, Bartholomev B (2002) High-resolution mapping of changes in histone-DNA contacts of nucleosomes remodeled by ISW2. Mol. Cell. Biol. 22:7524-34.

[45] Battaglioli E, Andres ME, Rose DW, Chenoweth JG, Rosenfeld MG, Anderson ME, MAndel G (2002) REST repression of neuronal genes requires components of the hSWI:SNF complex. J. Biol. Chem. 277:41038-45.

[46] Urnov FD (2002) Methylation and the Genome: the power of a small amendment. J. Nutr. 132:24506.

[47] Amar P et al. (2002) Hyperstructures, genome analysis and I-cell. Acta Biotheor. in the press.

[48] Hall IM, Shankaranarayana GD, Noma K, Ayoub N, Cohen A, Grewal SIS (2002) Establishment and maintenance of a heterochromatin domain. Science 297:2232.

[49] Chen T, Ueda Y, Xie S, Li E (2002) A novel Dnmt3a isoform produced from an alternative promoter localizes to euchromatin and its expression correlates with active de novo methylation. J. Biol. Chem. 277:38746-54.

[50] Brewer L, Corzett M, Balhorn R (2002) Codensation of DNA by spermatid basic nuclear proteins. J. Biol. Chem. 277:38895-900.

[51] Muchardt C, Seeler JS, Trouche D, Dejean A, Yaniv M (2002) Coordinated methyl and RNA binding is required for heterochromatin localization of mammalian HP1alpha. EMBO R. 3:975-84.

[52] Scheid OM, Probst AV, Afsar K, Paszkowski J (2002) Two regulatory levels of transcriptional gene silencing in Arabidopsis. Proc. Natl. Acad. Sci. 99:13659-62.

[53] Czermin B, Melfi R, McCabe D, Seitz V, Imhof A, Pirrotta V (2002) Drosophila enhancer of ZESTE/ESC complexes have a histone $\mathrm{H} 3$ methyltransferase activity that marks chromosomal polycomb sites. Cell 111:

[54] Muller J, Hart CM, Francis NJ, Vargas ML, Sengupta A, wild B, Miller EL, O'Connor MB, Kingston RE, Simon JA (2002) Histone methyltransferase activity of a drosophila polycomb group repressor complex. Cell 111:

[55] Mahmoudi T, Verrijzer CP (2001) Chromatin silencing and activation by polycomb and thrithorax group proteins. Oncogene 28:3055-66. 
[56] Labrador M, Corces VG (2002) Setting the boundaries of chromatin domains and nuclear organization. Cell 111:

[57] Bodnar JW (1988) A domain model for eukaryotic DNA organization: a molecular basis for cell differentiation and chromosome evolution. J. Theor. Biol. 132:479-507.

[58] Cremer T, Cremer C (2001) Chromosome territories, nuclear architecture and gene regulation in mammalian cells. Nat. Rev. Genet. 2:292-301.

[59] Santos-Rosa H, Schneider R, Bannister AJ, Sherriff J, Bernstein BE, Tolga Emre NC,Schreiber SL, Mellor J, Kouzarides T (2002) Active genes are tri-methylated at K4 of histone H3. Nature 419:40711.

[60] Lacoste N, Utley RT, Hunter JM, Poirier GG, Cote J (2002) Disruptor of telomeric silencing 1 is a chromatin-specific histone H3 methyltransferase. J. Biol. Chem. 277:30421-4.

[61] Smale ST, Fisher AG (2002) Chromatin structure and gene regulation in the immune system. Annu. Rev. Immunol. 20:427-62.

[62] Jackson DA (2002) Chromosome structure and nuclear architecture: implications for gene-therapy. Curr. Opin. Mol. Ther. 4:290-8.

[63] Jones PA, Baylin SB (2002) The fundamental role of epigenetic events in cancer. Nat. Rev. Genet. 3:415-28.

[64] Klenova E, Morse H, Ohlsson R, Lobanenkov V (2002) The novel BORIS+CTCF gene family is uniquely involved in the epigenetics of normal biology and cancer. Semin. Cancer Biol. 12:399.

[65] Li E (2002) Chromatin modification and epigenetic reprogramming in mammalian development. Nat. Rev. Genet. 3:662-73.

[66] Rajewsky N, Benecke A (2002) Genome-wide identification of a hematopoetic enhancer by exploiting the coregulator model of transcription. in preparation.

[67] Rajewsky N, Vergassola M, Gaul U, Siggia ED (2002) Computational detection of genomic regulatory modules, applied to body patterning in the early drosophila embryo. under revision.

[68] Rajewsky N, Zavolan M, Socci ND, Gaasterland T (2002) SMASHing regulatory sites in DNA by human-mouse sequence comparisons. submitted. 


\section{Legends to Figures:}

\section{Fig. 1:}

Transcription Regulation in Pro- and Eukaryotes.

A schematic presentation (not to scale) of transcription regulation in prokaryotes and eukaryotes illustrates the change in paradigm concerning template structure. While prokaryotic gene regulation is only little controlled via the structure of the DNA-protein complex, eukaryotes extensively use chromatin to repress and activate transcription of a gene. Thereby, the contribution of recruitment and modification of general transcription factors (GTF) becomes a relative minor contribution to overall transcription activity with respect to total required energy. Note also that while transcription regulation in prokaryotes can be approximated to a discrete process, in eukaryotes the wealth of chromatin and GTF modifications (compare Figure 2), the degeneration of the regulatory code, the increased number of interpretable signals per regulatory region, and the nonlinearities in signal processing allow continuous tuning of gene activity.

\section{Fig. 2:}

The Coregulator Concept in Eukaryotes, Functional Distance of Regulatory Elements.

Transcription factor (here: A, B, C) activity is a function of its functional distance to the transcription start site (general transcription factors (GTFs), ' +1 ') and the incoming signals it responds to. Functional distance does not necessarily reflect spacing along the linear DNA, but rather depends on the DNA template structure. Coregulators change the chromatin structure by reversible methylation $\left('-\mathrm{CH}_{3}{ }^{\prime}\right)$, acetylation ('-CO- $\left.\mathrm{CH}_{3}{ }^{\prime}\right)$, and phosphorylation $\left('-\mathrm{PO}_{3}{ }^{2-1}\right)$, regulate higher order chromatin packaging, and participate in the assembly and modification of general transcription factors and their cofactors, thereby either reducing (coactivator) or increasing (corepressor) this functional distance.

\section{Fig. 3:}

Coregulator induced Transcription Factor Synergy \& DNA Binding Cooperativity.

Upper Panel: The coregulator (CoR) induces synergy (defined as more than additive activity) between two transcription factors (A, B) when it can co-bind both proteins. By contrast, transcription activity 
between CoR-A and $B$ or $A$ and CoR-B, as measured by mutating (' $x$ ') the CoR for interaction with either one transcription factor, is only additive $[14,15]$.

Lower Panel: The DNA binding activity of a hetero-dimeric transcription factor complex (AB) is cooperatively increased in the presence of a specific CoR. While A can also form homo-dimeric complexes on the composite recognition sequence, B requires $A$ as a partner for binding [17].

Fig. 4:

Coregulator-Induced Synergy between transcription factors.

Coregulators might induce synergy between transcription factors by two distinct means. Concomitant binding to the participating transcription factors (upper panel, left) might be required to raise the local concentration of the coregulator and its time of engagement to the level where its activity exceeds a threshold for gene activation. The absence of any of the participating transcription factors will weaken the coregulator-transcription factor complex to the point where activity falls below the threshold (right side). Note, that the experimental demonstration of coregulator-induced synergy (Figure 3, [14, 15, 16]) described here most likely is best represented by this model. Alternatively, coregulator-induced synergy might be result of timely-related recruitment of several molecules of coregulator (identical or different proteins) by the participating transcription factors. Transcriptional activity of the target gene then rather depends on the establishment of a stable chromatin-modified domain over a minimal distance exceeding a spatial threshold (lower panel, left). The absence of any participating transcription factor will render the size of the resulting chromatin domain inferior to the minimal requirement (right side). Currently, no experimental method exists that could discriminate both possibilities in an in vivo system. Most likely, actual mechanisms of synergy are a merge of both options.

\section{Fig. 5:}

Activation-Repression Switching \& Transcription-DNA Repair crosstalk by Coregulators.

Upper Panel: CoR-1 acts as a corepressor on transcription factor A when A is mutated (' $x$ ') and unable to interact with CoR-2. CoR-2 displays no measurable activity when bound to A in the absence of CoR1. When both CoR-1, and CoR-2 can bind to A, CoR-2 switches CoR-1 from a corepressor to a coactivator $[18,19]$. 
Lower Panel: The specific coregulators CoR-1 and CoR-2 can both act as transcription coactivators for transcription factor A. They act synergistically when present together. However, CoR-1 and CoR-2 can, through cross-regulation of their associated enzymatic activities, turn DNA-repair competent. The implication of the CoR-1/CoR-2 complex in both transcription regulation and DNA repair strongly suggest functional coupling of both activities [20].

\section{Fig. 6:}

\section{The Secondary-Code Concept.}

Access of transcription factors to their cognate responsive elements (active binding sites) in chromatin can be considered as a hen-egg problematic. Current models of transcription activation require the transcription factor to recognize its target site before recruitment of coactivators which in turn will render the chromatin domain prone to transcription. The transcription factor thus orchestrates the initial step in gene activation through its specificity for the regulatory element. However, several documented cases show that a stable transcription factor-DNA complex is only formed upon activation of transcription, suggesting that the chromatin needs to be opened by a coactivator before or concomitant to engagement of the transcription factor with its binding site. This experimental evidence is conflicting with above models with respect to the required specificity. With the recent demonstration of chromatin-modifications being required for gene-activation [8] and the suggestion that chromatin-modifications along the nucleosomal array might constitute a secondary layer of gene regulatory coding [upper panel, sinus-curve represents nucleosomal array, see text] an alternate model is proposed: Coregulators might use the histonemodifications as 'flags' to identify on a genomic scale sites that are prone to transcription regulation (lower panel, step 1). These domains are then 'opened' by the coactivator in an indiscriminant fashion. Upon activation, the gene-specific transcription factor can access DNA only throughout all pre-opened domains, and stably engage at those sites where a recognition element is present. The transcription factor will then in turn further stabilize coregulator activity and orchestrate GTF recruitment (step 2). Sites that have been pre-opened in analogy constitute secondary recognition sites for corepressors which will reverse the nonspecific chromatin opening by the coactivator, thus establishing equilibrium between both adverse processes (chromatin breathing). The transcription factor, through its specific and stable engagement with regulatory elements will temporarily shift the equilibrium to the open-state at selected (specific) sites. The 
sequence of the different histone-modifications along the nucleosomal array thus might play a function analog to the sequence of nucleotides in regulatory regions (TF binding) by directing specific coregulator engagement with chromatin.

\section{Fig. 7:}

Chromatin-Code leads to hypercycles.

A model illustrating the concept of a secondary gene regulatory code carried by histone-modifications is depicted. Through the action of a coactivator ('CoA') a repressed ('[R]') nucleosome is, in an energy consuming process, converted to an open ('[O]') state (i.e. acetylation, methylation etc.). This state in-turn may allow a DNA-sequence specific transcription factor ('TF') to bind its target sequence and remove the nucleosome from the equilibrium-cycle between repressed and open states by converting it into an active site of transcription ('[A]'). Otherwise, the open state nucleosome is recognized as a substrate by a corepressor ('CoR') and converted back to the repressed state, thus establishing an energy driven hypercycle between the $[R]$ and $[\mathrm{O}]$ states. Finally, analogous to the action of a transcription factor on the [O] state, epigenetic regulatory factors ('ER') might remove [R] state nucleosomes from the hypercycle by converting them to condensed ('[C]') nucleosomes (i.e. higher order chromatin structures established after DNA-methylation). This model predicts that coactivators ('CoA') will open chromatin at all nucleosomal sites that are a substrate for the specific CoA enzymatic activity (low specificity), whether they are used as sites of gene regulation or not. Only in a subsequent step (high specificity), through the engagement of a transcription factor ('TF'), a subset of these opened sites become sites of active transcription ('[A]'), while the remainder is being converted back to the $[R]$ state through the action of a corepressor ('CoR') with opposite activity as the CoA. 


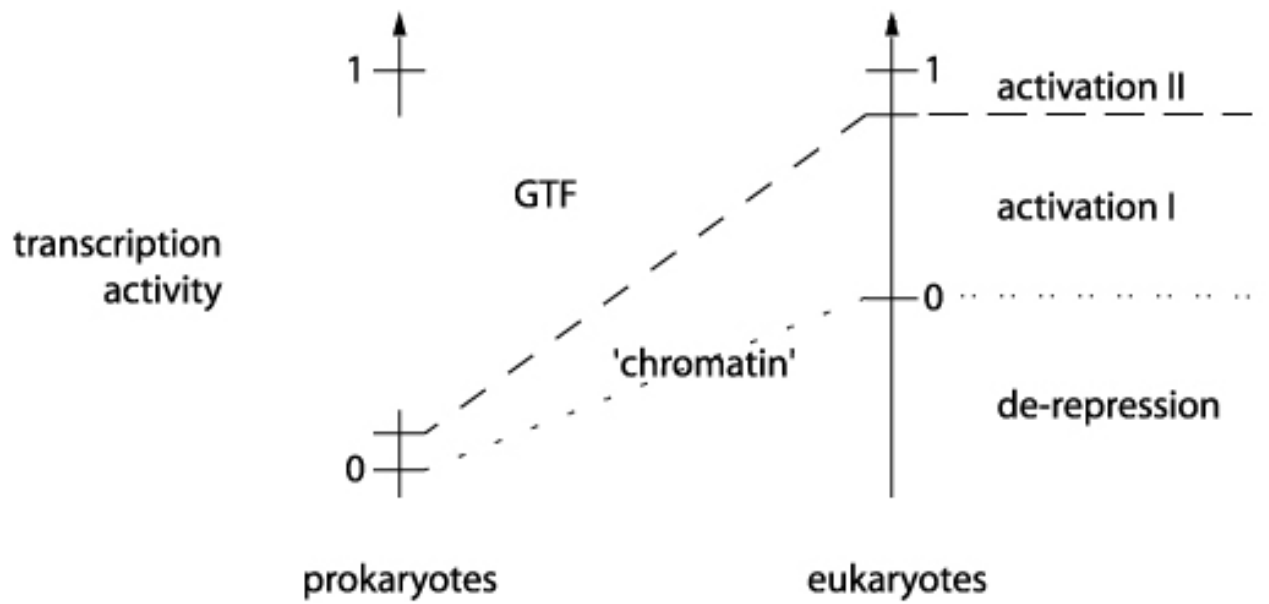

Benecke 2002, Figure 2

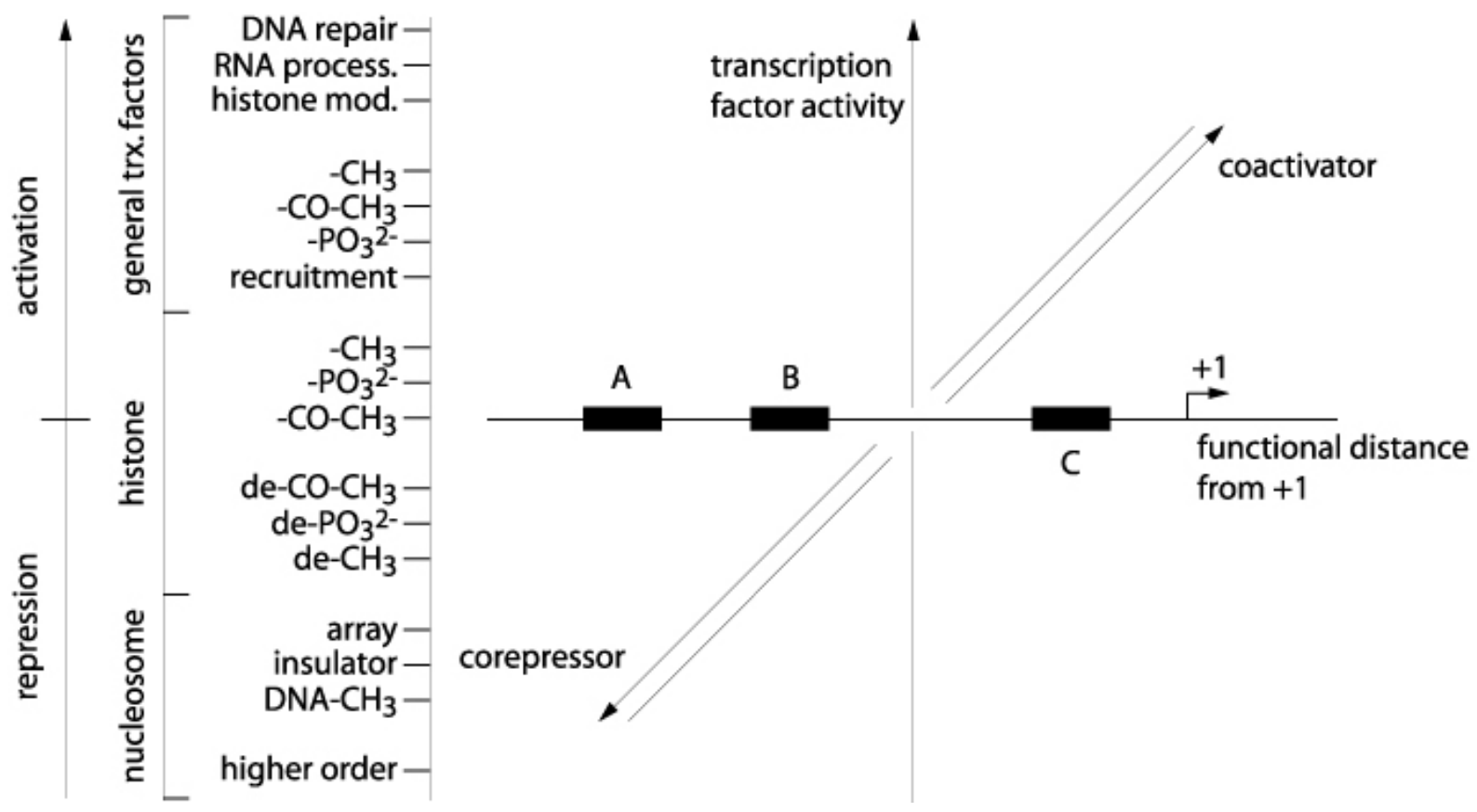


Benecke 2002, Figure 3
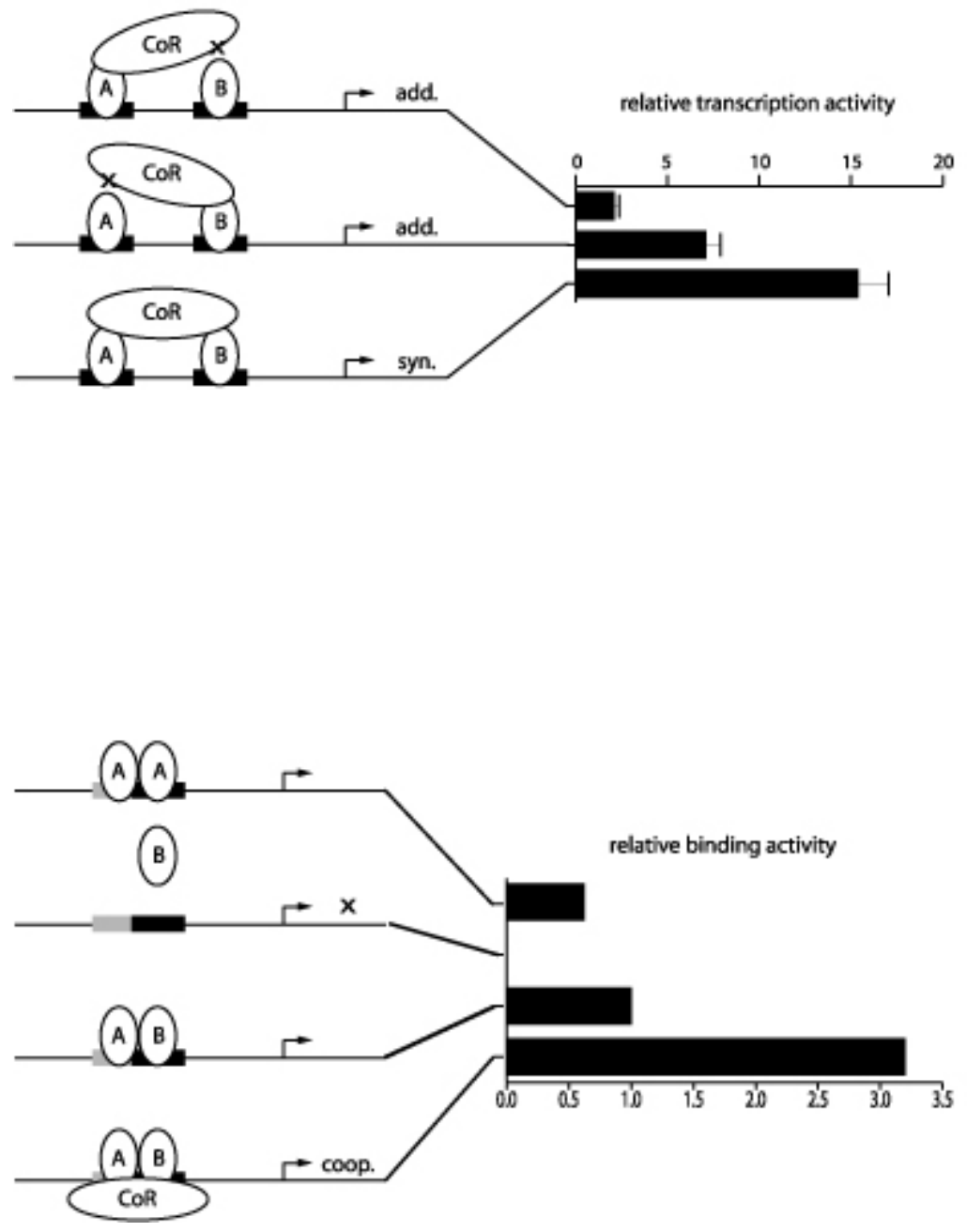
the coregulator model of transcription, arndt benecke

Benecke 2002, Figure 4
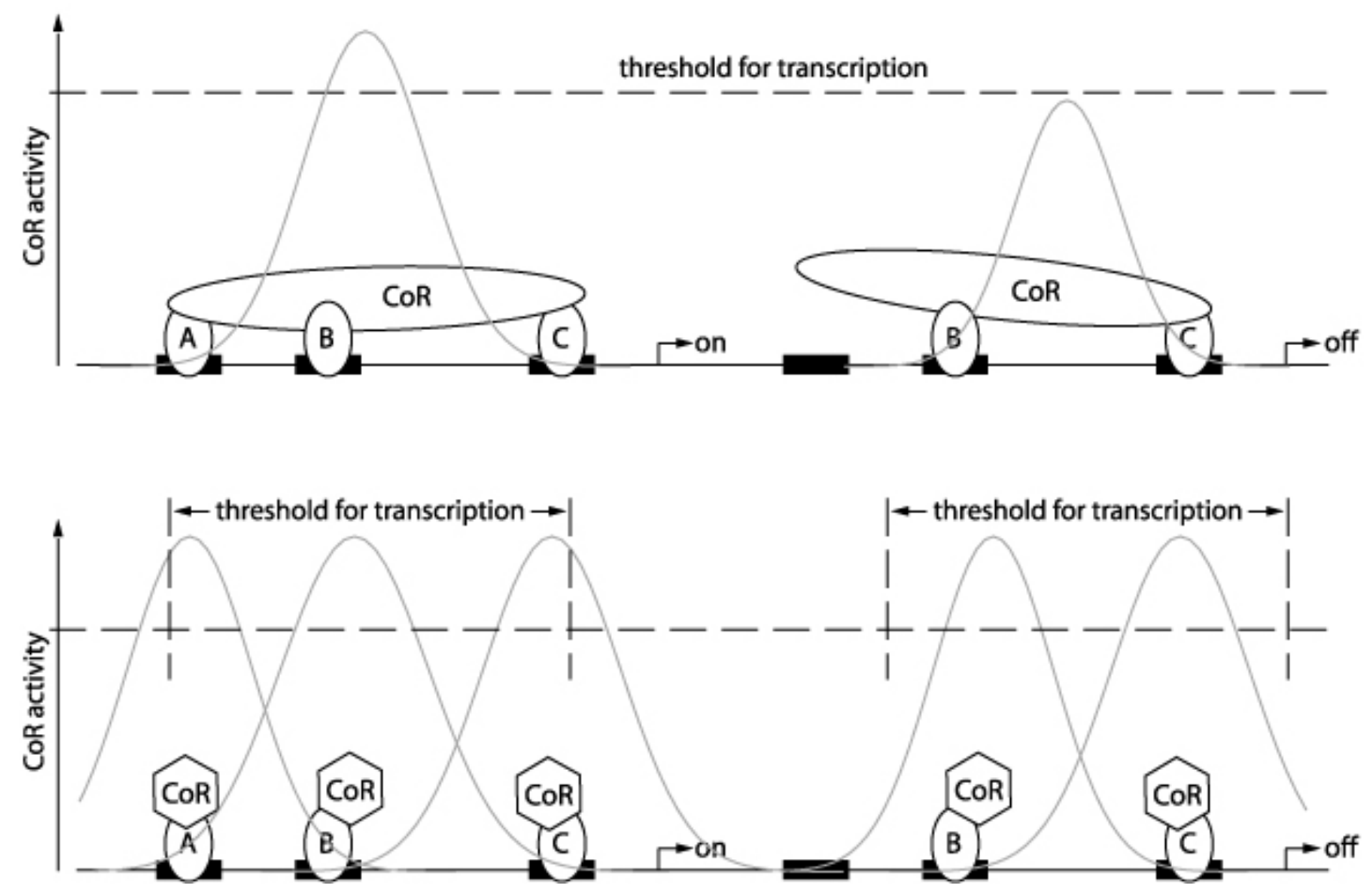

$28 / 31$ 


\section{Benecke 2002, Figure 5}
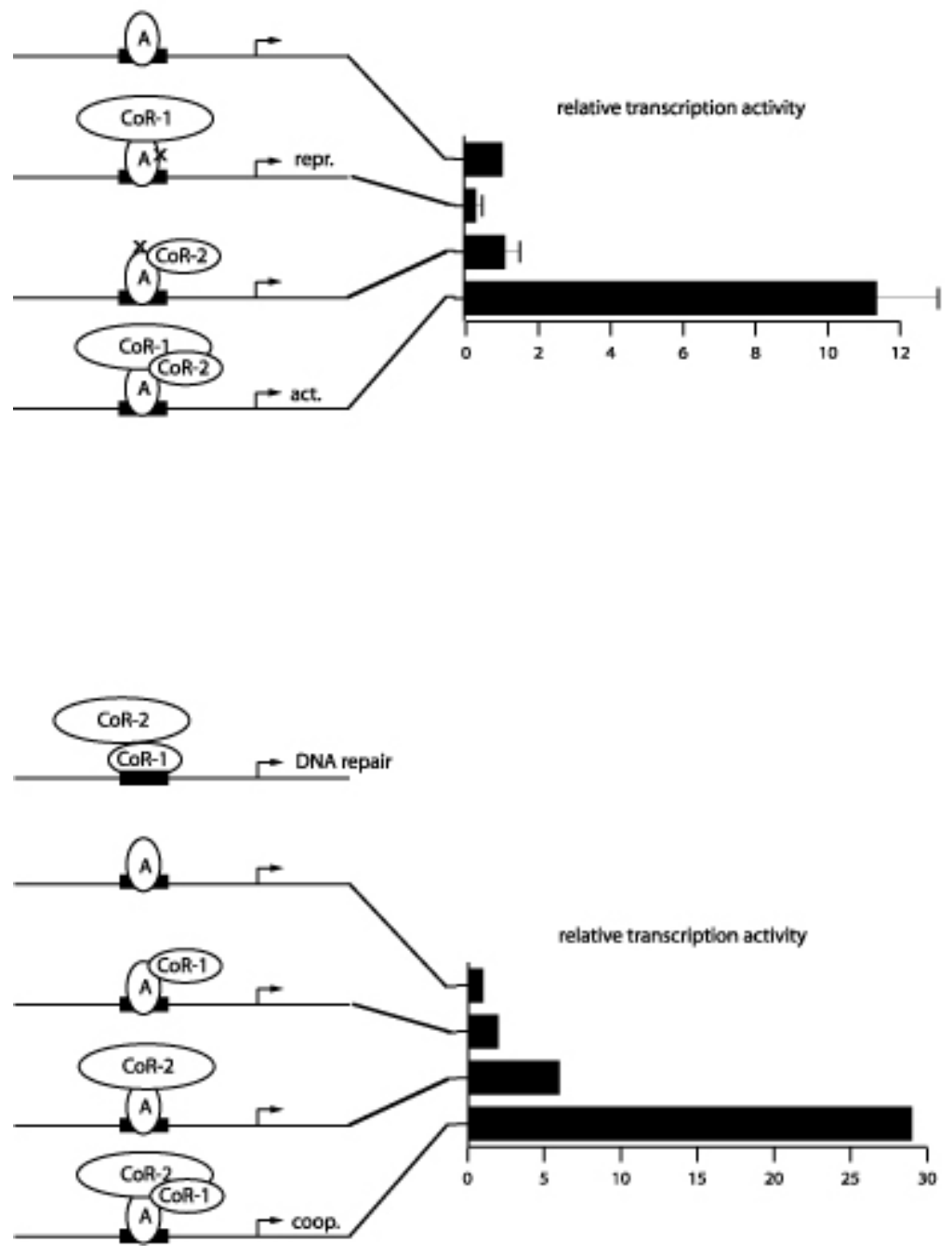


\section{Benecke 2002, Figure 6}

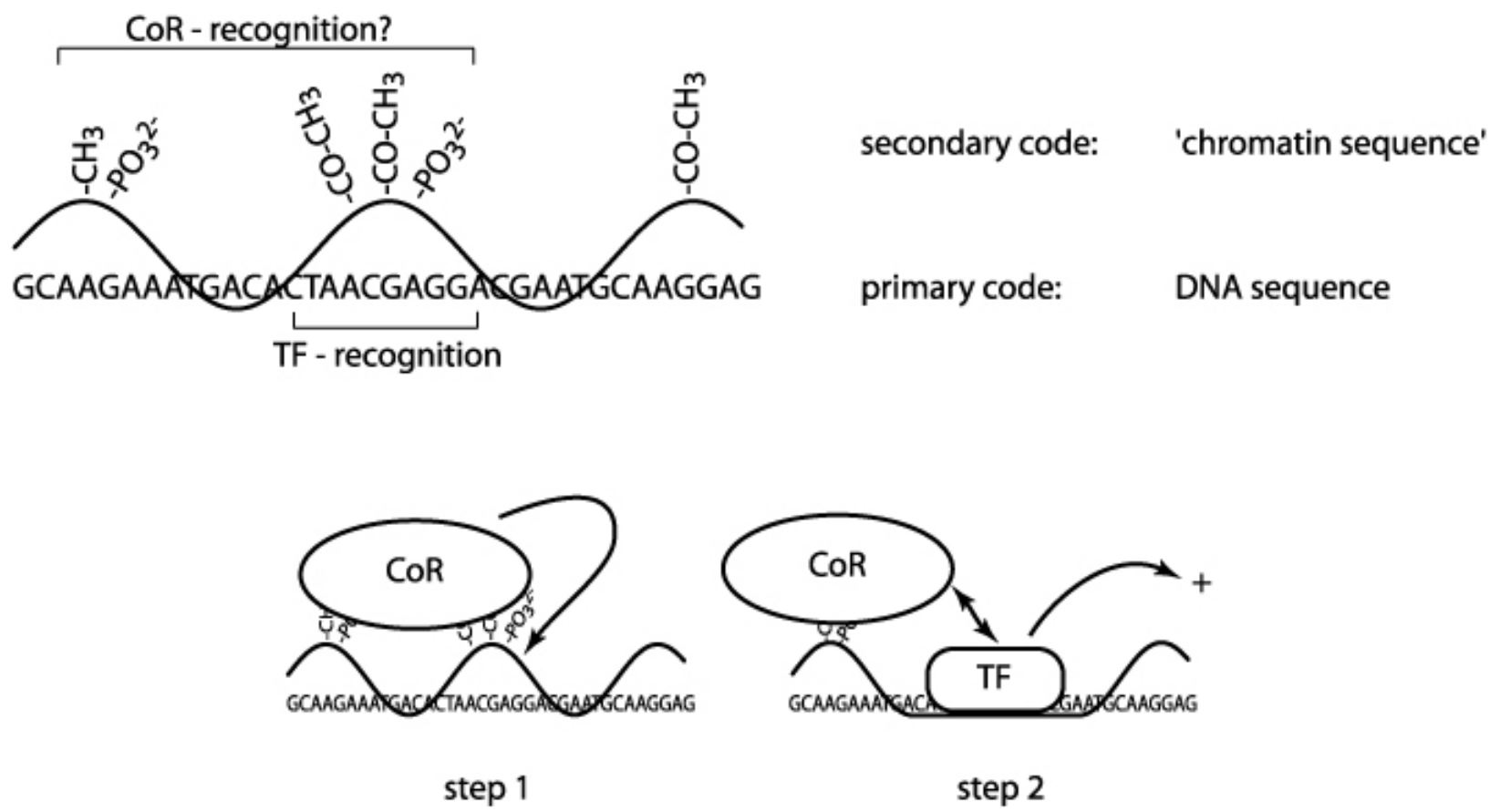

Benecke 2002 - Figure 7

heterochromatin

functional states

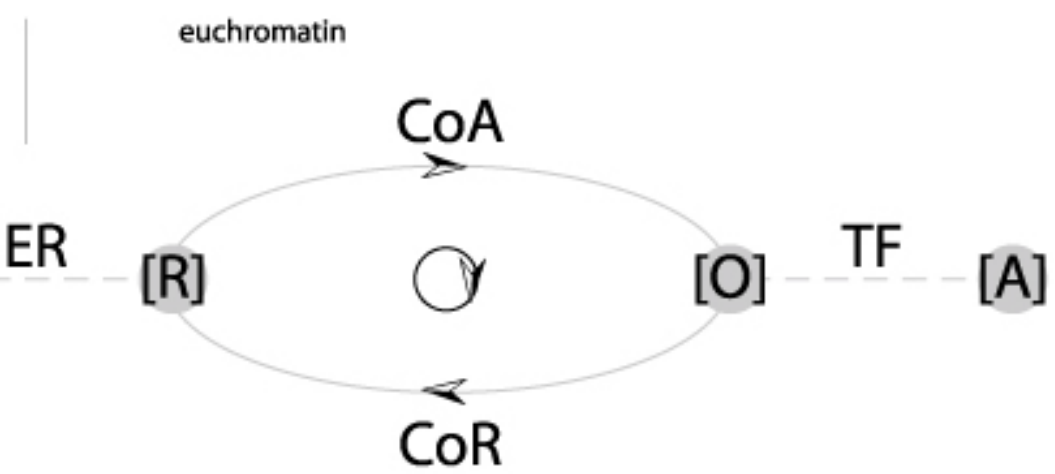

activity with respect to DNA-Seq, specific event 